\title{
Early Activation Markers of Human Peripheral Dendritic Cells
}

\author{
Peter Hellman and Håkan Eriksson
}

\begin{abstract}
Two major populations of dendritic cells (DCs), myeloid and plasmacytoid, can be isolated from human peripheral blood, and are distinguished by differential expression of the cell surface markers CD11c and CD123. These two populations of DCs also are different in their expression of Toll-like receptor (TLRs), which are involved in their activation. To investigate the early events during activation of peripheral DCs, the cells were stimulated in vitro with ligands for TLR-4 (as in lipopolysaccharides [LPS]) or TLR-9 (CpG-containing oligonucleotide $[\mathrm{CpG}])$. The earliest change in protein expression detected after stimulating peripheral DCs with lipopolysaccharide (LPS) or CpG was increased production of the chemokine interleukin (IL)-8. Enhanced production of IL-8 occurred already within 2 hours of stimulation in both myeloid dendritic cells (M-DCs) and plasmacytoid dendritic cells (P-DCs), and preceded expression of the well established activation marker CD40. Although both populations of DCs secreted IL-8 upon activation, the levels of IL-8 produced was several times higher within the M-DCs compared with the P-DCs population. Before
\end{abstract}

$\begin{array}{ll}\text { ABBREVIATIONS } \\ \text { CpG } & \text { CpG-containing oligonucleotide } \\ \text { DC } & \text { dendritic cell } \\ \text { IL } & \text { interleukin } \\ \text { LPS } & \text { lipopolysaccharide } \\ \text { M-DC } & \text { myeloid dendritic cell }\end{array}$

\section{INTRODUCTION}

Dendritic cells (DCs) play a central role in our immune system [1]. The DCs are the most important cell type responsible for antigen presentation, and as such they control both initiation and maintenance of adaptive immune responses, as well as induction of peripheral tolerance [2-5].

From the Department of Biomedical Laboratory Science, Health and Society, Malmö University, S-20506 Malmö, Sweden.

Address reprint requests to: Hakkan Eriksson, Department of Biomedical Laboratory Science, Health and Society, Malmö University, S-20506 Malmö, Sweden: Fax: +46406658100; E-mail: hakan.eriksson@bs. mah.se.

Received June 30, 2006; revised January 17, 2007; accepted January 17, 2007. activation, both subsets of DCs expressed the IL-8 receptor type B (CD128b); but after stimulation the IL-8 receptor was down-regulated in both populations of DCs. Increased expression of MHC class II molecules is generally regarded as an early activation marker of DCs. However, only the P-DCs showed a significant up-regulation of MHC class II after stimulation. The M-DC population up-regulated MHC class II without any prior activation; thus care should be taken using increased expression of MHC class II molecules as an early activation marker of peripheral M-DCs after activation in vitro.

In conclusion, we propose that during activation of human DCs the production of IL-8 and loss of CD128b are the earliest signs of activation preceding both $\mathrm{MHC}$ class II, CD40, CD80, and CD86 expression. Human Immunology 68, 324-333 (2007). (C) American Society for Histocompatibility and Immunogenetics, 2007. Published by Elsevier Inc.

KEYWORDS: CpG; LPS; MHC expression; IL-8; Myeloid dendritic cell; Plasmacytoid dendritic cell

PAMP pathogen-associated molecular pattern

P-DC plasmacytoid dendritic cell

PRR pattern recognition receptor

TLR toll-like receptor

Immature DCs are normally found in peripheral blood. Upon activation these cells will up-regulate surface molecules such as MHC class II, adhesion and co-stimulatory molecules, thereby adopting a mature phenotype [6]. The DCs sample and sense their environment and pass this information on to the immune system. When pathogenic organisms or substances are encountered, the presence of non-self material will immediately be communicated to the immune system. Pathogens are primarily recognized by a large number of pathogen-associated molecular patterns (PAMPs) that are identified by conserved pattern recognition receptors (PRRs), such as the Toll-like receptors (TLRs) [7]. Several TLRs have been identified and 
proven to be crititically involved in provoking maturation of DCs $[8,9]$.

In addition, TLRs are also differentially expressed on diverse DC subsets [9, 10]. For example, in human peripheral blood, the two major subpopulations of DCs, the myeloid (M-DCs) and the plasmacytoid (P-DCs) dendritic cells, differently express TLR4 and TLR9 $[9,10]$. The initial phenotypic changes that occur after DC activation are important and interesting to study to gain insight in the regulation of our immune system. However, the early events after myeloid and plasmacytoid DC activation remain relatively unknown. Here, we describe some early activation events at the protein level after in vitro stimulation of DCs using ligands to TLRs.

\section{MATERIALS AND METHODS}

\section{Cells and Reagents}

Peripheral blood buffy coat preparations were obtained from the Blood Bank of the University Hospital of Malmoe (Sweden). Blood dendritic isolation kit II (MACS), LD and MS columns were from Miltenyi Biotec, Bergisch Gladbach, Germany.

Lipopolysaccharide (LPS, from Escherichia coli O111:B4) was from Sigma (St. Louis, MO). The CpG oligodeoxynucleotide (ODN) sequences, $5^{\prime}$-tcgtcgttttgtcgttttgtcgtt- $3^{\prime}$ and 5'-ggGGGACGATCGTCgggggg 3', phosphorothioate (PTO) linkages between the bases are shown in lower case and phosphodiester linkages are shown in capital letters, were obtained from MWG-BIOTECH AG (Ebersberg, Germany). The ODNs were suspended in sterile $\mathrm{dH}_{2} \mathrm{O}$ and stored and handled under aseptic conditions. A Limulus Amebocyte Lysate (LAL) kit (Charles River Endosafe, Charles River Laboratories, Inc., Charleston, SC) was used to determine the amount of endotoxin in the $C_{p} G$ preparation and was shown to be, in $5 \mu \mathrm{g} / \mathrm{ml}$ solution of $\mathrm{CpG}$, below detection limit of the assay (0.5 ng LPS/ml).

N-hydroxysuccinimide biotin (NHS-biotin) was purchased from Bio-Rad Laboratories (Richmond, CA). RPMI 1640 with L-glutamine, gentamicin, Dulbecco's PBS without $\mathrm{Ca}^{2+}$ and $\mathrm{Mg}^{2+}$ and human serum "off the clot" Type AB, were all purchased from PAA Laboratories (Linz, Austria). Ficoll-Paque was purchased from Amersham Pharmacia Biotech AB (Uppsala, Sweden). Fragmin was obtained from Pfizer (Täby, Sweden). Human IgG (h-IgG) (Gammanorm) was purchased from Biovitrum AB (Stockholm, Sweden).

The BD Cytofix/Cytoperm Plus (with GolgiStop) cytokine intracellular staining, Cytometric Bead Array (CBA) human inflammation kit, Annexin V-FITC Apoptosis detection Kit I and 7-Amino-Actinomycin D (7-AAD) were all purchased from Becton Dickinson (San Diego, CA).

\section{Isolation of Human Peripheral Blood Dendritic Cells}

Peripheral blood mononuclear cells (PBMCs) were separated using peripheral blood buffy coat preparations from healthy donors by density-gradient centrifugation on Ficoll-Paque [11] with added Fragmin (20 units/ml).

Isolation of human peripheral DCs was performed by a two-step procedure using the human blood dendritic cell isolation kit II (MACS) using LD and MS columns following the manufacturer's instructions. Briefly, PBMC were incubated with non-DC depletion cocktail (containing biotin labeled anti BDCA-1 and microbeads conjugated with monoclonal antibodies against human CD14 and CD19) in PBS containing 0.5\% bovine serum albumin (BSA) and $2 \mathrm{mmol} / \mathrm{L}$ EDTA for 15 minutes at $4^{\circ} \mathrm{C}$, washed and applied on a MACS column. The flow through fraction containing pre-enriched DCs, were incubated with DC enrichment cocktail (microbeads conjugated with antibodies against biotin, human BDCA-4 and BDCA-3) in PBS containing $0.5 \% \mathrm{BSA}$ and 2 $\mathrm{mmol} / \mathrm{L}$ EDTA for 15 minutes at $4^{\circ} \mathrm{C}$ and washed once. Labeled dendritic cells were adsorbed on a MACS column and eluted after removal of the column from the magnetic device. To achieve highest purity of the DC population, the positively selected cells were after elution, separated over a second MACS column as a standard procedure.

\section{In Vitro Stimulation of Isolated Peripheral Blood Dendritic Cell Preparations}

Cells were suspended in culture medium; RPMI 1640 supplemented with $10 \%$ human serum "off the clot," Type $\mathrm{AB}$, and $100 \mu \mathrm{g} / \mathrm{ml}$ of gentamicin. Isolated dendritic cells were incubated at a final concentration of $1 \times$ $10^{6} / \mathrm{ml}$ in Eppendorf tubes rotated with short rotation intervals using a Triomix-rotator (Triolab AB, Sweden) in the presence or absence of LPS $1 \mu \mathrm{g} / \mathrm{ml}$ or $\mathrm{CPG}$ $5 \mu \mathrm{g} / \mathrm{ml}$.

The $\mathrm{CpG}$ sequences used in this study were $5^{\prime}$-tcgtcgttttgtcgttttgtcgtt- $3^{\prime}$, which corresponds to the B-class ODN-2006 [12] and 5'-ggGGGACGATCGTCgggggg 3', which corresponds to the A-class ODN 2216 [13], both used in previous studies. The CpG sequence of the B-class was used throughout in this paper except in experiments inducing production of $\alpha$-interferon where the $\mathrm{CpG}$ sequence of the A-class was used. All cells were incubated at $37^{\circ} \mathrm{C}$ in a humidified atmosphere containing $5 \% \mathrm{CO}_{2}$ for up to 8 hours. Stimulated or un-stimulated isolated dendritic cells were upon end of incubation tested for signs of necrosis or apoptosis using 7-AAD and Annexin-FITC. Briefly, according to the manufacturer's instruction, cells, final concentration $1 \times 10^{6} / \mathrm{ml}$, were washed twice in cold PBS and resuspended in $1 \times$ binding buffer, $5 \mu \mathrm{l}$ 
Annexin V-FITC and $0.5 \mu \mathrm{l}$ 7-ADD of supplied solution was added. The solution was then incubated in RT in the dark for 15 minutes. Finally cells were resuspended in $500 \mu \mathrm{l}$ of $1 \times$ Binding buffer, final volume, and analyzed by flow cytometry.

\section{Flow Cytometry}

Monoclonal antibodies used were FITC conjugated antiCD4 (MT30), FITC conjugated anti-CD11c (KB90) from DakoCytomation (Glostrup, Denmark). PE-conjugated anti-CD11c (B-ly6), PE-Cy5 conjugated anti-IL-8 Rec. B (CD128b) (6C6), PE-Cy5 conjugated anti-CD123 (9F5), PE-Cy5 conjugated anti-CD80 (L307.4) FITC conjugated anti-CD33 (HIM3-4), FITC conjugated antiCD86 (FUN-1), FITC conjugated anti-CD40 (5C3), and its recommended isotype control FITC conjugated (MOPC-21) were all purchased from Becton Dickinson (San Diego, CA). PE conjugated anti-HLA-DR, DQ (WR18) were obtained from Serotec (Oxford, United Kingdom). FITC conjugated anti-BDCA-2 (AC144), PE conjugated anti BDCA-4 (AD5-17F6), and FITC and PE conjugated anti-CD123 (AC145) were all purchased from Miltenyi Biotec (Bergisch Gladbach, Germany). Biotin conjugated anti-IL-8 Rec. A (CD128a) (NVR1) was obtained from Novus Biologicals Inc. (Littleton, CO).

Hybridomas producing antibodies against human MHC class I (Hb149) and MHC class II (Hb104) were obtained from ATCC/LGC-Promochem (Teddington, United Kingdom). After purification of the antibodies by proteinG-Sepharose, the antibodies were labeled with $\mathrm{N}$-hydroxysuccinimidebiotin [14].

Streptavidin-peridinin chlorophyll-a protein conjugate (SAV-PerCP) was purchased from Becton Dickinson (San Diego, CA).

Briefly, after incubation at $37^{\circ} \mathrm{C}$ or on ice, cells $(1 \times$ $10^{5}$ to $2 \times 10^{5}$ ) were washed with $1 \mathrm{ml}$ PBS containing $0.1 \%(\mathrm{w} / \mathrm{v}) \mathrm{BSA}$ (PBS-BSA), re-suspended in $100 \mu \mathrm{l} \mathrm{PBS}$ containing $0.1 \%(\mathrm{w} / \mathrm{v}) \mathrm{BSA}$ and $0.1 \%(\mathrm{w} / \mathrm{v}) \mathrm{h}-\mathrm{IgG}$ (PBS$\mathrm{BSA}-\mathrm{hIgG}$ ) and stained with fluorescent labeled primary antibodies for 30 minutes on ice in the dark. Finally the samples were washed with $1 \mathrm{ml}$ PBS-BSA, re-suspended in $500 \mu \mathrm{l}$ of PBS-BSA and analyzed by flow cytometry.

After incubation with biotin labeled antibodies the cells were washed with $1 \mathrm{ml}$ PBS-BSA, re-suspended in $100 \mu \mathrm{l}$ of PBS-BSA-hIgG containing SAV-PerCP, final dilution 1:400 and incubated for 30 minutes on ice in the dark. Finally the samples were washed with $1 \mathrm{ml}$ PBS-BSA, re-suspended in $500 \mu \mathrm{l}$ of PBS-BSA and analyzed by flow cytometry.

For intracellular staining, cells were stained for cell surface antigens, before the intracellular staining procedure. Briefly, cells $\left(1 \times 10^{6} / \mathrm{ml}\right.$, final concentration $)$ in medium or stimulated with LPS or CpG were incubated for $2,4,6$, or 8 hours. To accumulate intracellular IL-8,
GolgiStop was added to the cell incubations in a final dilution of 1:1500 2 hours before harvesting the cells and staining with antibodies. After harvesting, the cells $(1 \times$ $10^{5}$ to $2 \times 10^{5}$ ) were washed with PBS-BSA-hIgG, stained with fluorescent labeled antibodies against CD11c and CD123, permeabilized and fixed according to the manufacturers instructions (Becton Dickinson, San Diego, CA). Finally the cells were stained with PE conjugated antibodies against IL-8 at a final concentration of $0.5 \mu \mathrm{g} / \mathrm{ml}$ (G265-8, Becton Dickinson) and analyzed by flow cytometry.

Intracellular IL-8 receptor type B (CD128b) was analyzed 4 hours after incubation with LPS or CpG $37^{\circ} \mathrm{C}$. As a control, cells were incubated in medium at $37^{\circ} \mathrm{C}$ and at $4^{\circ} \mathrm{C}$. The cells were harvested, washed, stained for surface expression of CD11C and CD123, permeabilized, and fixed. Finally the cells were stained for intracellular $\mathrm{CD} 128 \mathrm{~b}$ and analyzed by flow cytometry.

Cells were analyzed by FACScan using standard settings and CELLQuest version 3.3 (Becton Dickinson).

\section{Detection of Secreted Cytokines}

Cytokine content in the medium after stimulation with CPG of the class B or LPS was assayed using the Cytometric Bead Array (CBA) human inflammation kit from BD Pharmingen/Becton Dickinson. Briefly, cell culture $\left(1 \times 10^{6} / \mathrm{ml}\right.$, final concentration) medium supernatants were collected upon end of an incubation period and stored at $-20^{\circ} \mathrm{C}$. The concentration of IL- 8 , IL- $1 \beta$, IL-6, IL-10, TNF- $\alpha$, and IL-12p70 were then determined in the supernatants $(50 \mu \mathrm{l})$ according to the manufacturer's instruction using a FACScan flow cytometer and BD Software (Becton Dickinson).

The content of $\alpha$-interferon was determined by an enzyme-linked immunosorbent assay kit purchased from PBL Biomedical Laboratories (Piscataway, NJ). In brief, collected supernatants $(100 \mu \mathrm{l}$ in duplicates and stored at $-20^{\circ} \mathrm{C}$ ) from $\mathrm{CpG}$-conditioned cell culture medium $\left(1 \times 10^{6} / \mathrm{ml}\right.$, final concentration) after 8 or 16 hours of stimulation were analyzed for $\alpha$-interferon using a human interferon- $\alpha$ enzyme linked immunosorbent assay kit according to the manufacturer's instruction. Results were analyzed with KC-Junior Software version 1.41.4 (Bio-tek Instruments, Winooski, Vermont).

\section{In Vitro Activation of DCs Before Isolation From PBMCs}

The PBMCs were suspended in culture medium. A 60-ml quantity of PBMCs at a final concentration of $1 \times$ $10^{7} / \mathrm{ml}$ was incubated 4 hours in two $50-\mathrm{ml}$ tubes in the presence or absence of LPS $10 \mu \mathrm{g} / \mathrm{ml}$ in a humidified atmosphere containing $5 \% \mathrm{CO}_{2}$ for 4 hours at $37^{\circ} \mathrm{C}$. During incubation the tubes were rotated using a MACS mix (Mx001, Miltenyi Biotec, Bergisch Gladbach, Ger- 
many). Upon end of incubation dendritic cells were isolated using the human blood dendritic cell isolation kit II (MACS) as previously described. Expression of CD40, MHC class II and intracellular accumulation of IL-8 by the isolated DCs were then analyzed by flow cytometry. For intracellular staining, cells were washed ones with PBS containing $0.1 \%$ (w/v) BSA (PBS-BSA) and then re-suspended in culture medium containing GolgiStop (final dilution of 1:1500) and left to incubate for 2 hours at $37^{\circ} \mathrm{C}$ in a humidified atmosphere containing $5 \% \mathrm{CO}_{2}$, before intracellular staining of IL-8 was performed. Before the intracellular staining procedure cells were stained with antibodies against CD11c and CD123 to identify the DC populations.

\section{RESULTS}

As previously described, after isolating DCs from human peripheral blood, two major populations of DCs could be identified. The preparations of DCs showed on average $56 \pm 13 \% \mathrm{CD} 11 \mathrm{c}^{+}$and $36 \pm 10 \% \mathrm{CD} 123^{+}$dendritic cells $(n=10)$ and these cells correspond to myeloid and plasmacytoid DC based on the differential expression of the $\mathrm{CD}$ markers $\mathrm{CD} 11 \mathrm{c}$ (M-DCs) and CD123 (P-DCs) [4]. The M-DC and the P-DC populations showed the phenotype $\mathrm{CD} 4^{+}, \mathrm{CD} 11^{+}, \mathrm{CD} 33^{+}$and $\mathrm{CD} 4^{+}$, $\mathrm{CD} 123^{+}, \mathrm{BDCA}_{2}{ }^{+}$, and BDCA $4^{+}$, respectively. To further verify the $\mathrm{CD} 123^{+}$cells as plasmacytoid DC, isolated DCs were stimulated with CpGs of the A and B class. A clear production of $\alpha$-interferon was observed by the cells after 16 hours of stimulation (Table 1), and it should also be stressed that in accordance with Tomoki et al. [13] a clear difference between $\mathrm{CPG}$ of the A and B class was observed. Very low production of $\alpha$-interferon was obtained after stimulation with $\mathrm{CpG}$ of the $\mathrm{B}$ class whereas the A class induced very low up-regulation of the co-stimulatory molecules CD40, CD80, and CD86.

The two subpopulations of human peripheral DCs have been shown to differentially express TLRs important for the activation of DCs. The M-DC population express TLR-4 while the P-DCs express TLR-9 [9, 10].

TABLE 1 Secretion of $\alpha$-interferon by isolated human peripheral dendritic cells after stimulation with $\mathrm{CPG}$ of class $\mathrm{A}$ and $\mathrm{B}$

\begin{tabular}{cccc}
\hline & \multicolumn{3}{c}{ Concentration $\alpha$-interferon $(\mathrm{pg} / \mathrm{ml})$} \\
\cline { 2 - 4 } $\begin{array}{l}\text { Stimulation } \\
\text { time (hours) }\end{array}$ & Medium control & CpG class A & CpG class B \\
\hline 16 & 23 & $>8,500$ & 140 \\
\hline
\end{tabular}

Abbreviations: $\mathrm{CpG}=\mathrm{CpG}$-containing oligonucleotide.

Purified dendritic cells (DCs) $\left(1 \times 10^{6} / \mathrm{ml}\right.$, final concentration) were stimulated with $5 \mu \mathrm{g} \mathrm{CpG} / \mathrm{ml}$. After 16 hours at $37^{\circ} \mathrm{C}$ the cells were removed and the content of $\alpha$-interferon in the medium was analyzed.
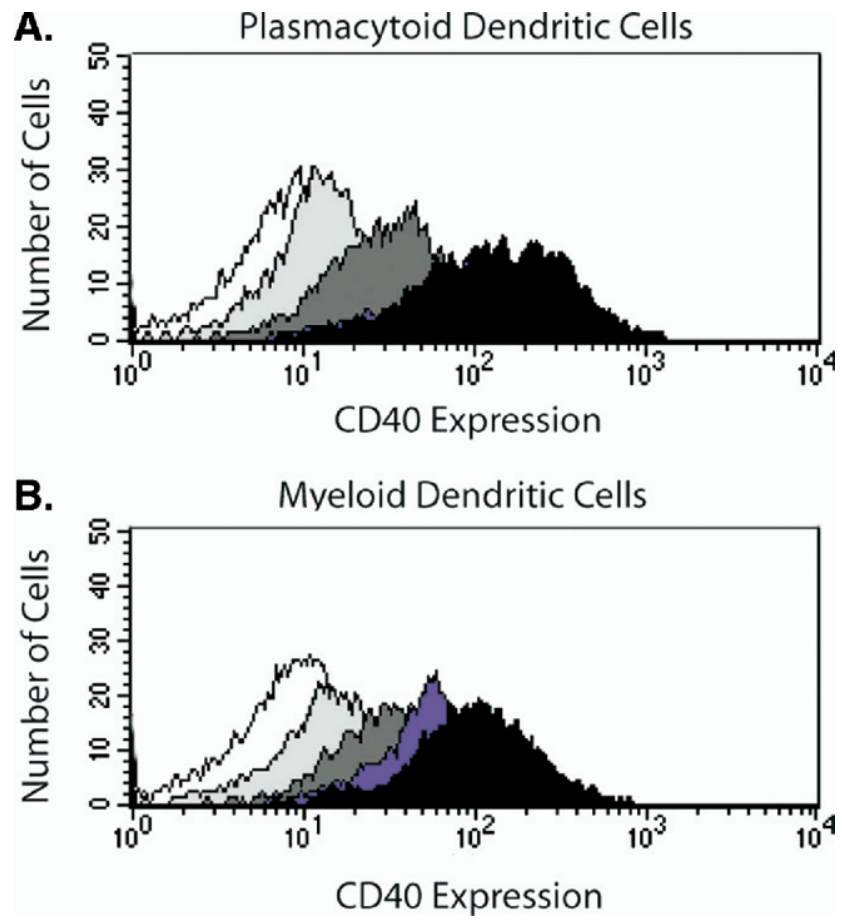

FIGURE 1 CD40 expression after in vitro activation. Isolated dendritic cells were stimulated in culture medium for up to 8 hours at $37^{\circ} \mathrm{C}$ with $\mathrm{CpG}$ or LPS. At indicated time periods the cells were harvest, washed, and stained with antibodies against CD123, CD11c, and CD 40. CD 40 ${ }^{+}$cells were distinguished based on a relevant isotype control after incubation at $4^{\circ} \mathrm{C}$. The histograms show from the left to right isotype control (white curve), cells stimulated for 2 hours (light-gray curve), 4 hours (medium-gray curve), 6 hours (dark-gray curve) and 8 hours (black curve) in culture medium at $37^{\circ} \mathrm{C}$. (A) Gated on P-DCs after CpG stimulation. (B) Gated on M-DCs after LPS stimulation. The figures show one representative experiment out of three.

To follow the early activation in peripheral DCs, the cells were stimulated in vitro with ligands for TLR-4 (LPS) or TLR-9 (CpG). As expected, the stimulation with LPS and $\mathrm{CpG}$ induced up-regulation of CD40, CD86 and CD80 in myeloid and plasmacytoid DCs, respectively. Expression of CD40 was used to verify activation of the DC populations (Figures 1 and 2) and was clearly upregulated in the $\mathrm{P}-\mathrm{DC}$ population after stimulation with CpG. No or almost no up-regulation of CD40 in the P-DC population was observed with LPS (Figure 2A). Although the M-DC population has not been reported to express TLR-9 [8], there was an unexpected upregulation of $\mathrm{CD} 40$ after incubation with $\mathrm{CpG}$ in the M-DCs population (Figure 2B) However, compared with stimulation with LPS, the up-regulation of CD40 was delayed by approximately 2 hours after incubation with CpG (Figure 2B).

Activation of peripheral DCs with LPS or CpG of the $\mathrm{B}$-class also resulted in production of several cytokines 


\section{A. Plasmacytoid Dendritic Cells}

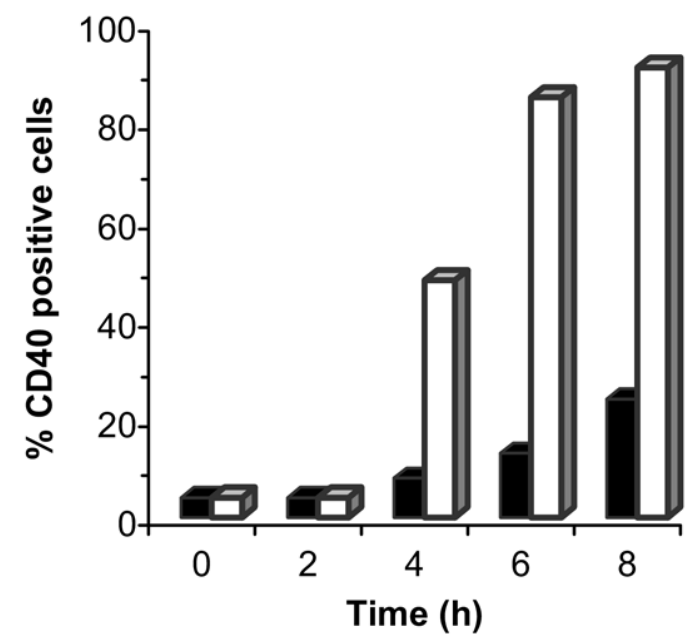

\section{B. Myeloid Dendritic Cells}

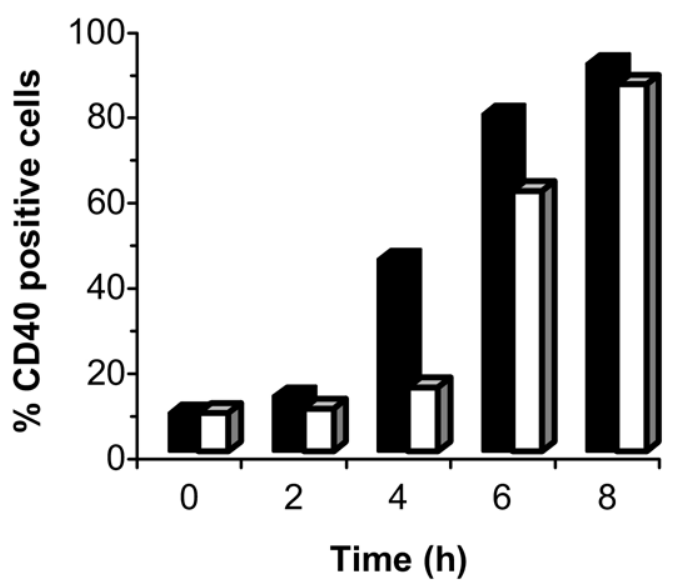

FIGURE $2 \mathrm{CD} 40^{+}$DCs after activation. Cells incubated in culture medium were stimulated with LPS ( $\square$ ) or CpG ( $\square$ ) for up to 8 hours at $37^{\circ} \mathrm{C}$. At 2-hour intervals samples were withdrawn, the cells were harvested, washed and stained with antibodies against CD11c, CD123, and CD 40. The CD $40^{+}$ cells were distinguished based on a relevant isotype control after incubation at $4^{\circ} \mathrm{C}$. (A) Gated on P-DCs. (B) Gated on M-DCs. The figures show one representative experiment out of three.

(Table 2). TNF- $\alpha$, IL-1b, IL-6, IL-8, IL-10, and IL12 p70 were analyzed and both LPS and CpG induced secretion of TNF- $\alpha$, IL-6, and high amounts of IL-8. An almost identical secretion of IL- 6 was obtained by both PAMPs and compared with stimulation using CpG, stimulation with LPS resulted in higher production of IL-8. On the other hand, stimulation with CpG caused a clearly enhanced production of TNF- $\alpha$, compared with LPS (Table 2).
Incubation with LPS or CpG of the B-class rapidly induced production of IL-8. The cellular production of IL-8 was analyzed by intracellular staining of the DC populations. Depending on the type of stimuli used, a clear difference between the M-DCs and the P-DCs in the amount of intracellular IL-8 accumulated was observed (Figure 3). The kinetic production of IL-8 by the two DC populations was investigated by arresting the secretion of IL-8 using GolgiStop for 2 hours at various times after activation. The P-DCs responded only to stimulation with $\mathrm{CpG}$ and after 4 hours a maximal production of IL-8 was reached (Figure 4A). The M-DCs produced IL-8 after stimulation with both of LPS or CpG (Figure 4B). However, the production of IL-8 after incubation with $\mathrm{CpG}$, was delayed by 2 hours, similar to the delayed expression of CD40 as described above (Figures $2 \mathrm{~B}$ and $4 \mathrm{~B}$ ). Almost all of the M-DCs produced IL-8 (80\%-90\% of the cells) after stimulation with LPS, whereas only $40 \%-50 \%$ of the P-DCs produced IL-8 after incubation with $\mathrm{CpG}$.

Before activation with LPS or CpG both subsets of DCs weakly expressed the IL-8 receptor type B (CD128b). However, upon stimulation, the IL-8 receptor was down-regulated and became undetectable in P-DCs and mearly detectable in M-DCs (Figure 5). Intracellular staining of non-stimulated and stimulated DCs was also performed, but the IL-8 receptor could not be detected intracellular in either DC population.

Increased surface expression of MHC class II are generally regarded as an early activation marker of DCs. Stimulation of the P-DCs with CpG of the B class induced a clearly higher up-regulation of MHC class II compared with stimulation by LPS (Figure 6A). Surprisingly, the M-DC population rapidly up-regulated MHC

TABLE 2 Cytokine secretion in isolated human peripheral dendritic cells after stimulation with LPS or CpG

\begin{tabular}{llrrrrr} 
& & \multicolumn{5}{c}{ Time (hours) } \\
\cline { 3 - 7 } Stimulation & $\begin{array}{c}\text { Cytokine } \\
(\mathrm{pg} / \mathrm{ml})\end{array}$ & \multicolumn{1}{c}{0} & \multicolumn{6}{c}{2} & \multicolumn{1}{c}{4} & 6 & \multicolumn{1}{c}{8} \\
\hline LPS & IL-8 & 7 & 514 & 2929 & $>5000$ & $>5000$ \\
& IL-6 & 3 & 5 & 71 & 142 & 235 \\
\multirow{4}{*}{ CpG } & TNF- $\alpha$ & 3 & 13 & 61 & 44 & 38 \\
& IL-8 & 11 & 131 & 1676 & 3184 & 3866 \\
& IL-6 & 5 & 4 & 63 & 139 & 174 \\
& TNF- $\alpha$ & 3 & 195 & 458 & 473 & 368 \\
\hline
\end{tabular}

Abbreviations: $\mathrm{CpG}=\mathrm{CpG}$-containing oligonucleotide; $\mathrm{LPS}=$ lipopolysaccharide; $\mathrm{IL}=$ interleukin; $\mathrm{TNF}=$ tumor necrosis factor.

Purified dendritic cells $\left(1 \times 10^{6} / \mathrm{ml}\right.$, final concentration) were stimulated with $1 \mu \mathrm{g} \mathrm{LPS} / \mathrm{ml}$ or $5 \mu \mathrm{g} \mathrm{CpG} / \mathrm{ml}$. After the indicated time points at $37^{\circ} \mathrm{C}$ the cells were removed and the cytokine content in the medium was analyzed. The presence of TNF- $\alpha$, IL-1b, IL-6, IL-8, IL-10, and IL-12p70 were assayed. The cytokine concentration as $\mathrm{pg} / \mathrm{ml}$ of one representative experiment of three is shown.

${ }^{a}$ IL-1b, IL-10, and IL-12p70, were analyzed and not detected. 
A.

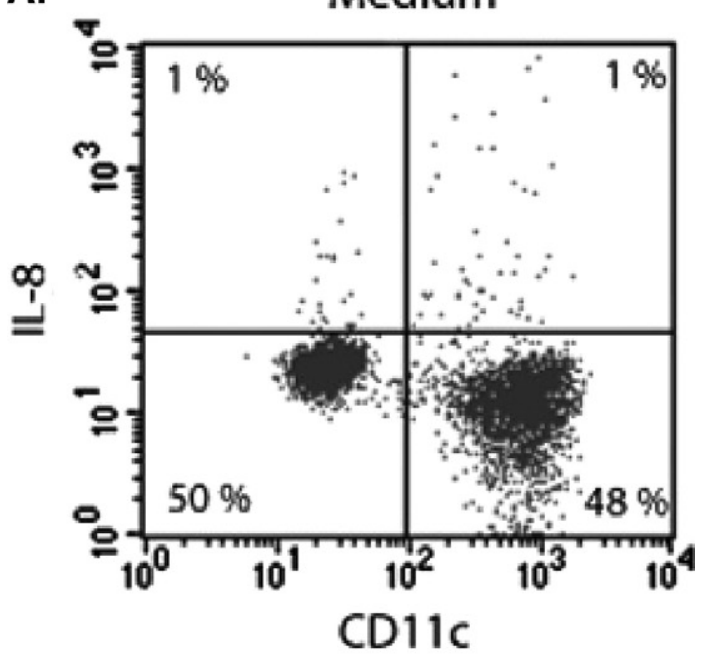

B.
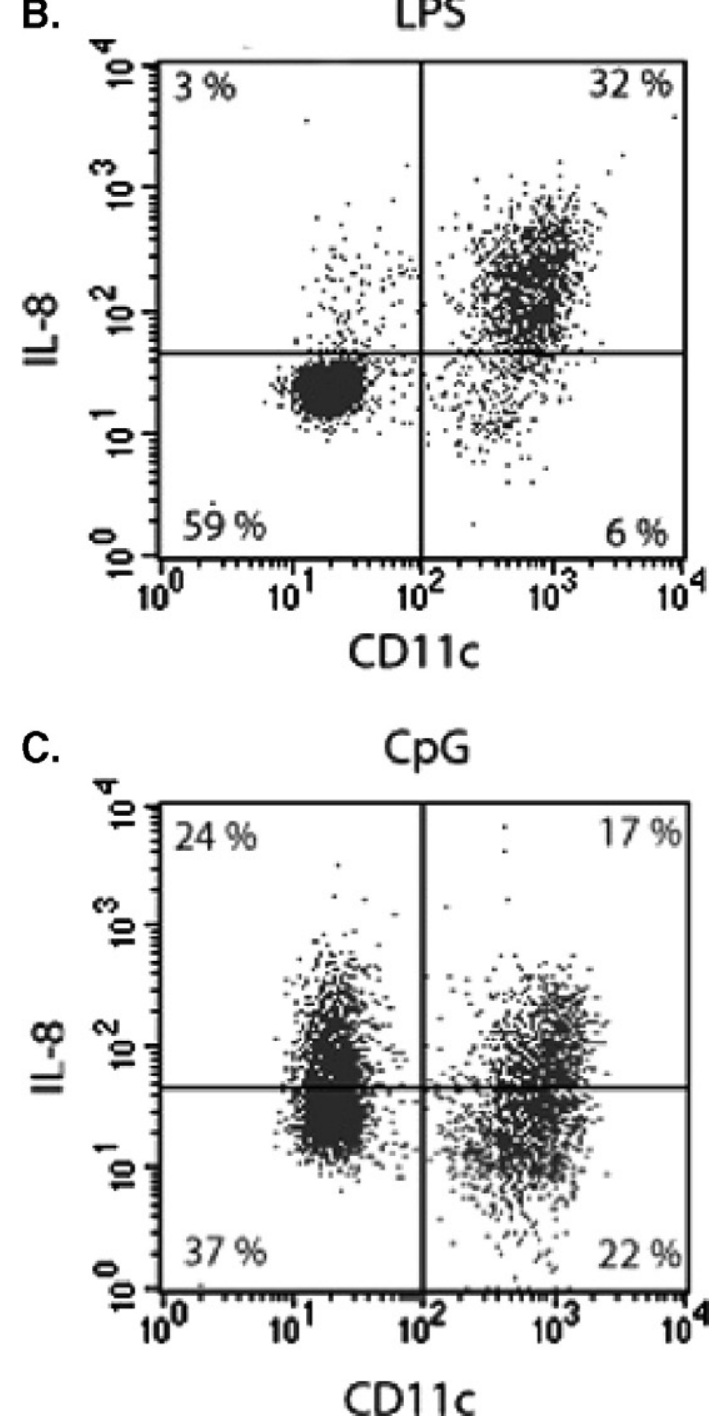

class II after conditioning in medium at $37^{\circ} \mathrm{C}$. This up-regulation was almost identical to the up-regulation after stimulation with PAMPs (Figure 6B and Table 3). No up-regulation of MHC class II was observed within the P-DCs population after conditioning in medium and no significant up-regulation of MHC class I was observed in either of the DC populations (Figure 6A and Table 3).

To verify our observed early activation events of DCs in the presence of other cells, PBMCs were stimulated with LPS for 4 hours before DCs were isolated using magnetic separation (MACS). DCs isolated from PBMCs stimulated with LPS showed the same activation markers as seen after stimulation of purified DCs. Briefly, upregulation of CD40 and high level of intracellular IL-8 by the M-DC population. The expression of MHC class II was equal to what was observed on M-DCs isolated from PBMCs incubated in control medium, confirming an early MHC-II expression independent of PAMP stimulation by the M-DC population.

\section{DISCUSSION}

The early events after peripheral DC activation are not well investigated. Plasticity and crossregulation during DC maturation events makes it important to understand and then in the future be able to regulate these events so that the final effector function of the dendritic cells can be controlled. This could perhaps generate more effective vaccines for treating infectious diseases or controlling and maintaining immune tolerance, or even providing us with effective methods for treating tumours.

Stimulation of purified peripheral DCs by CpG or LPS resulted in an up-regulation of the co-stimulatory molecules CD40 (Figures 1 and 2), CD86 and CD80 (data not shown). CD40 was the earliest up-regulated costimulatory molecule and was used to verify activation of the DC populations. LPS and CpG involve pathogenassociated molecular patterns and mediate activation of DCs by binding to different TLRs expressed by the DCs. Up-regulation of co-stimulatory molecules on P-DCs after stimulation with $\mathrm{CpG}$, and on M-DCs after stimulation with LPS was expected and correspond well with the reported expression of TLR-9 by P-DCs and TLR-4

FIGURE 3 Production of IL- 8 by activated DCs after 4 hours of stimulation. Dendritic cells (DCs) were incubated in culture medium at $37^{\circ} \mathrm{C}$ in the presence of LPS or CPG. After 4 hours the cells were harvest, washed and stained for the surface antigens CD123 and CD11c before intracellular staining for IL-8. Two hours before harvesting, GolgiStop (monensin) was added to the culture medium. (A) DCs in culture medium at $4^{\circ} \mathrm{C}$; (B) DCs stimulated with LPS; and (C) DCs stimulated with $\mathrm{CpG}$ in culture medium at $37^{\circ} \mathrm{C}$ for 4 hours. The figures show one representative experiment out of three. 


\section{A. Plasmacytoid Dendritic Cells}

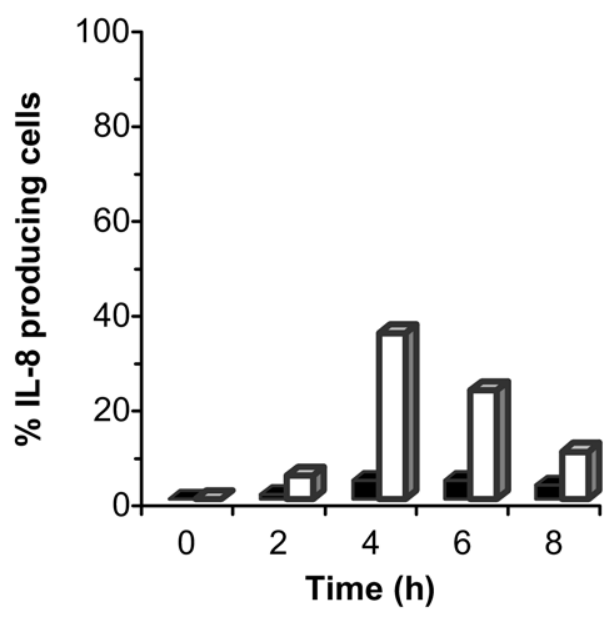

\section{B. Myeloid Dendritic Cells}

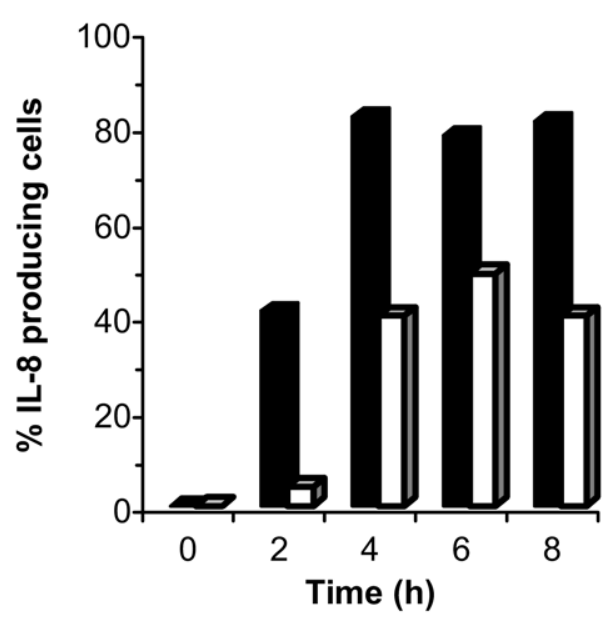

FIGURE 4 Dendritic cells producing IL-8 after activation. Isolated DCs were incubated in culture medium in the presence of CpG or LPS. At indicated time (0, 2, 4, 6, and 8 hours) after activation by CpG or LPS, produced IL-8 was accumulated intracellular for 2 hours by the addition of GolgiStop (monensin). The cells were then harvest and stained with antibodies against cell surface antigens, CD11c and CD123, before intracellular staining of IL-8. DCs incubated at $4^{\circ} \mathrm{C}$ were used as negative control. (A) P-DC after stimulation with CpG ( $\square$ ) or LPS ( $\square$ ) and (B) M-DC after stimulation with CpG ( $\square$ ) or LPS ( $\square$ ). The figures show one representative experiment out of three.

by M-DCs [9, 10]. Although some reports have indicated no or low expression of TLR-4 by M-DCs [15], our results showed that there is a clear up-regulation of co-stimulatory molecules after stimulation with LPS within this population.

P-DCs has not been reported to express TLR-4 [10]. In the presence of LPS, a weak up-regulation of co-stimulatory molecules by the P-DCs was obtained (Figure 2A). However, this up-regulation was similar when the P-DCs were incubated in medium (data not shown), suggesting that LPS does not induce activation of these cells.

Similarly, M-DCs have not been shown to express TLR-9 [8]. However, the cells showed a clear up-regulation of co-stimulatory molecules after incubating the purified DC preparation with $\mathrm{CpG}$ (Figure 2B). Stimulation of the DCs with CpG resulted in a slower kinetic expression of CD40 by the M-DC population compared with the P-DCs, and the M-DCs after stimulation with LPS (Figure 2).

Activation of P-DCs through TLR-9 induced secretion of TNF- $\alpha$ into the medium that was detected already after 2 hours of stimulation (Table 2). The M-DC population express TNF- $\alpha$ receptors of the type TNF- $\alpha$ RII [16], and TNF- $\alpha$ released by activated P-DCs may stimulate and

\section{A. Myeloid Dendritic Cells}

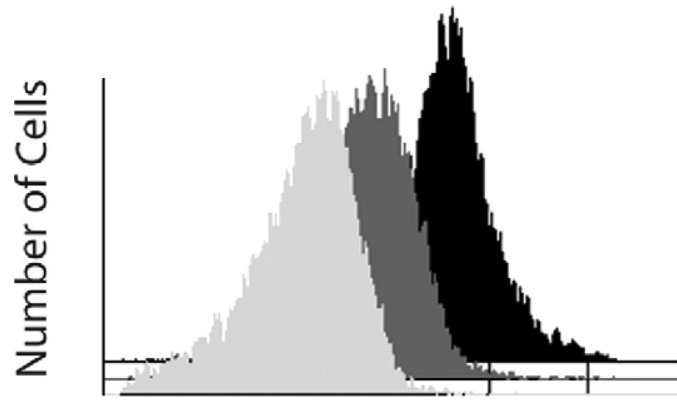

IL-8 Receptor Expression

B. Plasmacytoid Dendritic Cells

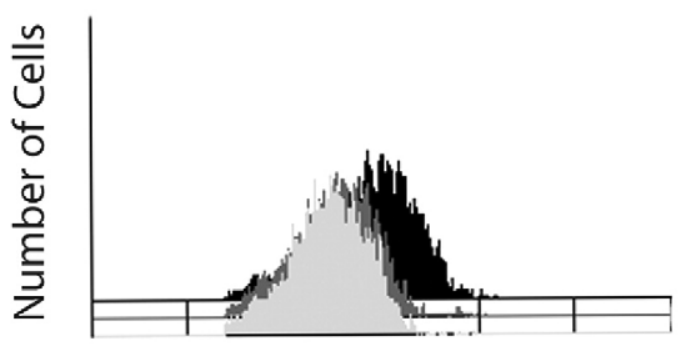

IL-8 Receptor Expression

FIGURE 5 Interleukin (IL)-8 receptor expression by DCs. Isolated DCs were incubated in culture medium in the presence or absence of $\mathrm{CpG}$ or LPS. After 4 hours at $37^{\circ} \mathrm{C}$ the cells were harvest and stained with antibodies against CD11c, $\mathrm{CD} 123$, and the $\mathrm{IL}-8$ receptor $(\mathrm{CD} 128 \mathrm{~b})$ or a relevant isotype control. DC populations were identified by flow cytometry and expression of the IL-8 receptor was analyzed. (A) M-DCs after incubation in medium (dark-gray curve), after stimulation with LPS (medium-dark-gray curve) and isotype control (light-gray curve). (B) P-DCs after incubation in medium (dark-gray curve), after stimulation with $\mathrm{CpG}$ (medium darkgray curve) and isotype control (light-gray curve). The figures show one representative experiment out of three. 
A. Plasmacytoid Dendritic Cells

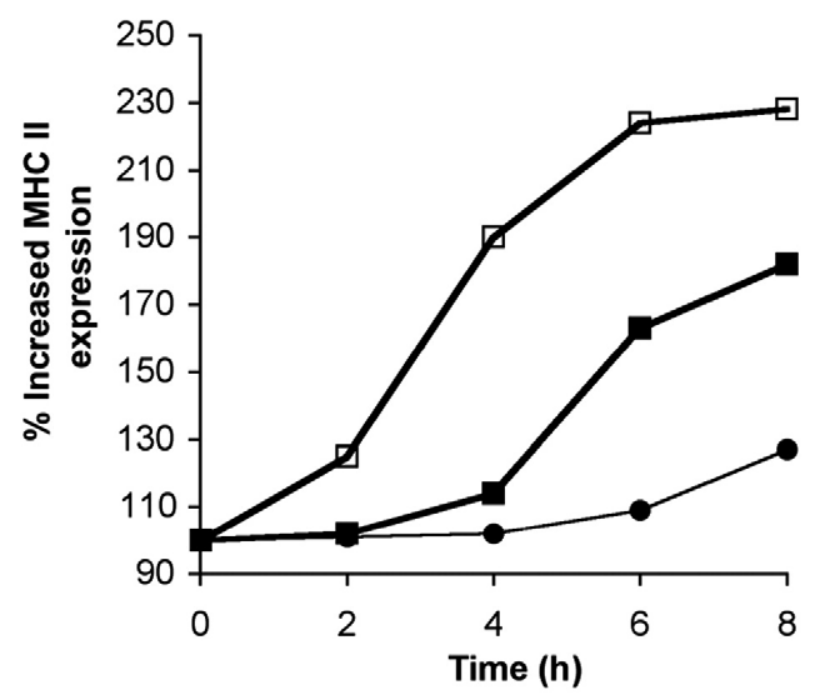

B. Myeloid Dendritic Cells

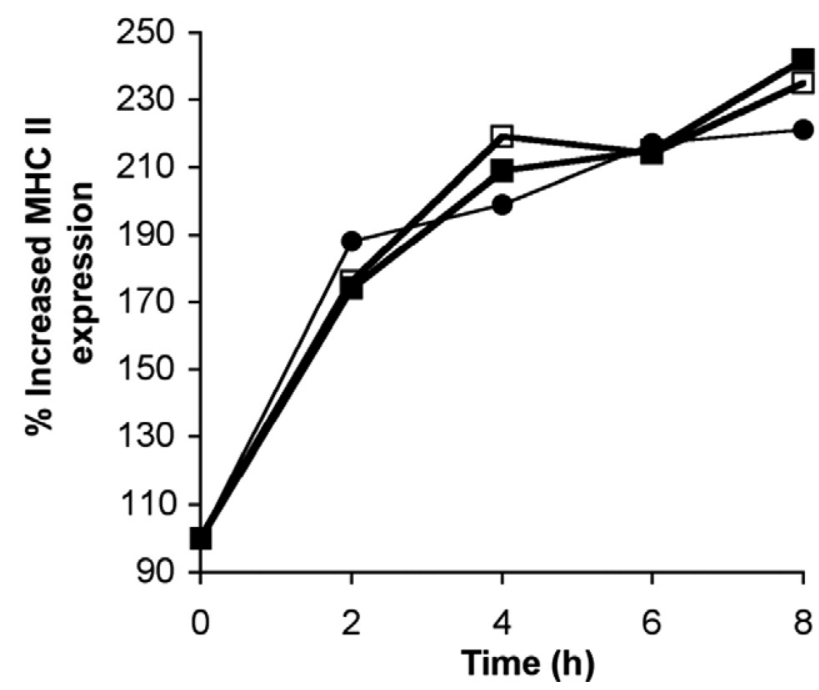

FIGURE 6 MHC class II expression by DCs after activation. Isolated DCs were incubated for up to 8 hours in culture medium ( or in the presence of LPS $(\square)$ or CpG $(\square)$. At 2-hour intervals samples were withdrawn, washed and stained with antibodies in order to identify the P-DC and M-DC population and their MHC II expression. MHC class II expression was determined as MFI values and the MFI value of nonstimulated cells incubated at $4{ }^{\circ} \mathrm{C}$ was set as $100 \%$. (A) MHC class II expression by P-DCs. (B) MHC class II expression by M-DCs. The figures show one representative experiment out of three.

activate the M-DC population. This could explain the observed up-regulation of co-stimulatory molecules by the M-DC population after stimulation with $\mathrm{CpG}$ and also their slower kinetic expression of CD 40.

Production of IL- 8 by activated DCs has been reported earlier $[17,18]$, and a major release of IL-8 into the medium was observed after stimulation of purified DCs with CPG or LPS (Table 2). Although both PAMPs induced high amounts of IL-8 in the medium, LPS induced much more IL-8 than did CPG. Intracellular staining of IL-8 revealed $80 \%-90 \%$ of the M-DCs to produce IL-8 after activation with LPS whereas only $40 \%-50 \%$ of the P-DCs produced IL-8 after activation with $\mathrm{CPG}$ (Figure 4). Although the preparations of DCs contained a lower amount of P-DCs compared with M-DCs, the reduced number of P-DCs producing IL-8 is probably the major explanation of the lower amount of IL-8 detected in the medium after stimulation with CpG.

Intracellular staining for IL-8 was performed on cells after accumulation of produced IL- 8 during a period of 2 hours at various times after activation. The highest number of DCs producing IL- 8 was seen after 4 hours of activation for both M-DCs and P-DCs. However, the number of P-DCs producing IL-8 peaked after 4 hours of activation (Figure 4A), whereas the M-DCs showed a more or less steady state in the number of cells producing IL-8 already after 4 hours of activation (Figure 4B).

PAMP induced production of IL-8 showed a faster kinetic rate than the corresponding up-regulation of co-stimulatory molecules and was observed already after 2 hours of stimulation with CPG or LPS (Figure 4). TLR- 4 is expressed both intracellular and in the cell

TABLE 3 Surface expression of MHC class I and II by dendritic cells after stimulation with LPS or CPG

\begin{tabular}{ccc}
\hline & \multicolumn{2}{c}{ MFI $37^{\circ} \mathrm{C} / \mathrm{MFI} 4^{\circ} \mathrm{C}$} \\
\cline { 2 - 3 } & Myeloid dendritic cells & Plasmacytoid cells \\
\hline MHC class II & $1.56 \pm 0.31$ & $1.06 \pm 0.24$ \\
Medium control & $(n=10)$ & $(n=10)$ \\
In presence of LPS & $1.60 \pm 0.32$ & $1.08 \pm 0.23$ \\
& $(n=10)$ & $(n=10)$ \\
In presence of CPG & $1.66 \pm 0.34$ & $1.73 \pm 0.30$ \\
MHC class I & $(n=6)$ & $(n=6)$ \\
Medium control & $1.03 \pm 0.07$ & $1.18 \pm 0.15$ \\
In presence of LPS & $(n=9)$ & $(n=9)$ \\
In presence of CPG & $1.15 \pm 0.15$ & $1.39 \pm 0.26$ \\
& $(n=9)$ & $(n=9)$ \\
& $(n=7)$ & $1.38 \pm 0.34$ \\
& $(n=7)$
\end{tabular}

Abbreviations: $\mathrm{CpG}=\mathrm{CpG}$-containing oligonucleotide; $\mathrm{DC}=$ dendritic cell; LPS = lipopolysaccharide; MFI = mean fluorescence intensity.

Results are presented as mean \pm SD. Myeloid and plasmacytoid dendritic cells (DC) were identified by flow cytometry using antibodies against CD11c and CD123. Expression of MHC class I and class II by M-DCs and P-DCs were analysed and the mean fluorescence intensity (MFI) of the cell populations were determined. MFI values were determined after; incubation in medium, stimulation with $1 \mu \mathrm{g} \mathrm{LPS} / \mathrm{ml}$ or stimulation with $5 \mu \mathrm{g} \mathrm{CpG/ml}$. The MFI ratios were calculated between cells incubated at $37^{\circ} \mathrm{C}$ for 4 hours and cells incubated in medium at $4^{\circ} \mathrm{C}$. 
membrane whereas TLR-9 is an intracellular receptor. The different localization of the receptors does not seem to affect the activation rate of the DC populations, because the production of IL-8 and up-regulation of costimulatory molecules occurred with similar kinetics in both populations. A recent paper has also shown the importance of endocytosed LPS in the activation process of M-DCs [19].

The production of IL- 8 by the M-DCs after stimulation in the presence of $\mathrm{CpG}$ showed a similar kinetic pattern as the up-regulation of their co-stimulatory molecules. This further strengthens the hypothesis that activation of the M-DC population after incubation with CpG is mediated by TNF- $\alpha$ or any other secondary stimulatory molecule produced by activated P-DCs. However, it should be noted that although 80\%-90\% of the M-DCs up-regulated their expression of CD 40 after incubation with $\mathrm{CpG}$ (Figure 2B), only about $50 \%$ of the M-DCs produced IL-8 after incubation with CpG (Figure 4B). This may well reflect a different signalling pathway and response mechanism by the M-DCs after stimulation by a PAMP mediated danger signal as LPS compared with an inflammation signal such as TNF- $\alpha$ [19].

To investigate a possible autocrine function of IL-8, the expression of IL- 8 receptors type A and B by the DCs were investigated (Figure 5). Only a weak expression of IL-8 receptor type B (CD128b) was observed on the surface of un-stimulated DCs $[18]$ and the expression was quickly down-regulated after stimulation with LPS or CpG. Since no up-regulation of the receptor and no intracellular receptor could be detected, our results do not support any autocrine function of IL-8 produced after activation of the DC populations. Although a conformation change of the receptor after binding IL-8 resulting in the disappearance of the epitope recognized by the antibody cannot be ruled out. However, the downregulation of surface expression of CD128b by the DCs could be used as an early sign of in vitro activation of peripheral DCs.

Up-regulation of MHC class II is generally considered as an activation marker of DCs and as expected, the P-DCs showed an increased expression of MHC class II after activation with $\mathrm{CPG}$ compared with incubation with LPS (Figure 6A and Table 3). The M-DC population however, up-regulated MHC class II regardless of stimulation, even by merely conditioning in medium (Figure 6B and Table 3).

P-DCs have an overall lower expression of MHC class II molecules compared with M-DCs [4] and the P-DCs have been shown to differ from all other DC subsets in their regulation of MHC class II gene expression [20]. This may have a functional consequence regarding the upregulation of MHC class II molecules between P-DCs and
M-DCs during the first hours after PAMP mediated activation, and our results clearly show that up-regulation of MHC class II cannot be used as an early marker of TLR mediated in vitro activation of peripheral M-DCs. The preparation of DCs, using positive selection with magnetic beads, may induce some form of activation of the M-DC population. However, the antibodies used in the purification process have not been reported to have any substantial effect on the isolated cells $[21]$ and our results agrees with a report by O'Doherty et al., showing a $\mathrm{CD} 11 \mathrm{c}^{+}$subset of DCs, obtained by negative panning, up-regulating their MHC class II after culture in medium for 36 hours [22].

Incubation of PBMCs with LPS before isolation of DCs verified the early activation events observed using purified DCs. In conclusion activation of peripheral blood DCs with PAMPs induce the production of IL-8 already within 2 hours of stimulation. The production of IL-8 preceded the up-regulation of the activation marker CD40 in both M-DCs and P-DCs and was several hours before any secretion of $\alpha$-interferon was observed into the medium by the P-DCs. Disappearance of the IL-8 receptor type B (CD128b) and production of IL-8 were the first changes in protein expression that were observed during the activation process of peripheral DCs.

Up-regulation of MHC class II molecules in response to TLR mediated activation were only observed by the P-DC population. The M-DC population showed an up-regulation of MHC class II without any prior activation, and care should be taken using increased expression of MHC class II molecules as an early in vitro activation marker of peripheral M-DCs.

\section{REFERENCES}

1. Banchereau J, Steinman RM: Dendritic cells and the control of immunity. Nature 392:245, 1998.

2. Liu Y-J, Kanzler H, Soumelis V, Gilliet M: Dendritic cell lineage, plasticity and cross-regulation. Nat Immunol 2:585, 2001.

3. Colonna M, Trinchieri G, Liu Y-J: Plasmacytoid cells in immunity. Nat Immunol 5:1219, 2004.

4. Rossi M, Young JW: Human dendritic cells: potential antigen-presenting cells at the crossroads of innate and adaptive immunity. J Immnol 175:1373, 2005.

5. Sousa CR: Dendritic cells in a mature age. Nat Rev Immunol 6:476, 2006.

6. Hart DNJ: Dendritic cells: unique leukocyte populations which control the primary immune response. Blood 90: 3245, 1997.

7. Akira S, Takeda K: Toll-like receptor signalling. Nat Rev Immunol 4:499, 2004. 
8. Loré K, Betts MR, Brenchley JM, Kuruppu JK, Khojasteh S, Perfetto S, Roederer M, Seder RA, Koup RA: Toll-like receptor ligands modulate dendritic cells to augment cytomegalovirus- and HIV-1-specific T-cell responses. J Immunol 171:4320, 2003.

9. Matsumoto M, Funami K, Tanabe M, Oshiumi H, Shingai M, Seto Y, Yamamoto A, Seya T: Subcellular localization of Toll-like receptor 3 in human dendritic cells. J Immunol 171:3154, 2003.

10. Hornung V, Rothenfusser S, Britsch S, Krug A, Jahrsdörfer B, Giese T, Endres S, Hartmann G: Quantitative expression of Toll-like receptor 1-10 mRNA in cellular subsets of human peripheral blood mononuclear cells and sensitivity to $\mathrm{CpG}$ oligodeoxynucleotides. J Immunol 168: 4531, 2002.

11. Böyum A: Separation of leukocytes from blood and bone marrow with special reference to factors which influence and modify sedimentation properties of hematopoietic cells. Scand J Clin Lab Invest 21 (Suppl):97, 1968.

12. Rothenfusser S, Hornung V, Ayyoub M, Britsch S, Towarowski A, Krug A, Sarris A, Lubenow N, Speiser D, Endres S, Hartmann G: CpG-A and CpG-B oligonucleotides differentially enhance human peptide-specific primary and memory $\mathrm{CD}^{+}{ }^{+} \mathrm{T}$-cell response in vitro. Blood 103:2162, 2004.

13. Tomoki I, Kanzler H, Duramad O, Cao W, Liu Y-J: Specialization, kinetics and repertoire of type 1 interferon responses by human plasmacytoid predendritic cells. Blood 107:6, 2006.

14. Harlow E, Lane D: Antibodies: A Laboratory Manual. Cold Spring Harbor, NY: Cold Spring Harbor Laboratory, 1988. p. 341.

15. Jarrossay D, Napolitani G, Colonna M, Sallusto F, Lanzavecchia A: Specialization and complementarity in microbial molecule recognition by human myeloid and plasmacytoid dendritic cells. Eur J Immunol 31:3388, 2001.

16. Gomez J, Borras FE, Singh R, Rajananthanan P, English N, Knight SC, Navarrete CV: Differential up-regulation of HLA-DM, invariant chain, and CD83 on myeloid and plasmacytoid dendritic cells from peripheral blood. Tissue Antigens 63:149, 2004.

17. Almeida J, Bueno C, Alguero MC, Sanchez ML, Canizo MC, Fernandez ME, Valquero JM, Laso FJ, Escribano L, San Miguel JF, Orfao A: Extensive characterization of the immunephenotype and pattern of cytokine production by distinct subpopulations of normal human peripheral blood $\mathrm{MHC} \mathrm{II}^{+} /$lineage ${ }^{-}$cells. Clin Exp Immunol 118: 392, 1999.

18. Penna G, Vulcano M, Sozzani S, Adorini L: Differential behavior and chemokine production by myeloid and plasmacytoid dendritic cells. Hum Immunol 63:1164, 2002.

19. Blander JM, Medzhitov R: Toll-dependent selection of microbial antigens for presentation by dendritic cells. Nature 440:808, 2006.

20. LeibundGut-Landmann S, Waldburger J-M, Sousa CR, Acha-Orbea H, Reith W: MHC class II expression is differentially regulated in plasmacytoid and conventional dendritic cells. Nat Immunol 5:899, 2004.

21. Dzionek A, Inagaki Y, Okawa K, Nagafune J, Röck J, Sohma Y, Winkels G, Zysk M, Yamaguchi Y, Schmitz J: Plasmacytoid dendritic cells: from specific surface marker to specific cellular functions. Hum Immunol 63:1133, 2002.

22. O'Doherty U, Peng M, Gezetler S, Swiggard WJ, Betjes M, Bhardwaj N, Steinman RM: Human blood contained two subsets of dendritic cells, one immunologically mature and the other immature. Immunology 82:487, 1994. 